with a new generation of larger, faster ferries (some with speeds over 50 knots) due to enter service shortly. Alongside the proposed changes there is also a need for electronics manufacturers to address the particular needs of the fast-ferry industry in order to enhance the electronic navigation capabilities of fast ferries.

KEY WORDS

I. Fast ferries. 2. Electronic charts. 3. Radar. 4. Pilotage.

\title{
Reduction to Final Speed: A Simple Formula
}

\section{Adrian Burnett}

In a recent issue of the Journal ${ }^{1}$ Captain Fales discusses the question of achieving an accurate arrival time concomitant with fuel economy. He proposes a method of monitoring permutations of speed by the setting up of simultaneous equations. Whilst agreeing with him, I believe the following alternative presentation of the problem makes the computation more intuitively appealing, and considerably more accessible and therefore useful to the average operator.

Firstly, the question of differentials in operating speeds extends beyond simple fuel economy to include ships with combination propulsion systems, typically warships. The considerate navigator of such a ship will co-operate with his engineer to plan passages in such a way that life cycles of gas turbines, as well as fuel economy, are taken into account. Even a short passage can present an alarming combination of possibilities; therefore the simpler the sums, the more satisfactory the result. Although graphing this sort of problem is useful, the results are never accurate enough, and they have to be reworked by another method to achieve precision.

Warship navigators also face another problem unknown to the merchant navigator namely that of getting a large and varied group of ships through pilotage waters and into harbour in some degree of order. Although the mathematics of this are identical to that of the single ship, the activity level involved in managing a formed body of ships is high, and the consequences of a mistake are embarrassing and public, especially when 'showing the flag' abroad. Again therefore, the most blunder-proof possible approach is indicated.

Simple though simultaneous equations may be at the classroom desk, they change from Jekyll into Hyde when the navigator is trying to write night orders for the formation commander at 2200 on a busy bridge. Is $x$ the higher or lower speed? Shall I allow for tidal stream and if so, where? How much slack do I want? These are all questions which beset the navigator at a time of pressure. The chances are that, once he has got a result by simultaneous equations, he will not feel inclined to re-work the whole thing to check it or, if he does, he will make the same mistake again. Even more importantly, the humble Officer of the Watch, entrusted with overseeing the night orders which will ensure successful arrival the next day, will be so put off by simultaneous equations that he will evade conscientious checking and monitoring, and will rely on the navigator's brilliance to carry things through. This belief in the navigator's infallibility usually presages a disaster of far-reaching proportions. However, given the simple formula 
below, cross-checks and re-works are easy, and even the mathematical rabbits will produce successful and painless arrivals.

The formula is derived from one in air navigation, designed to tell the aviator when it is time to turn round and go home. However, it has just as much use for the marine navigator. It is :

$$
t=\frac{D-H T}{O-H}
$$

where: $t=$ the elapsed time from when the calculation is done to when the reduction to terminal speed is ordered

$D=$ distance in nautical miles from calculation point to final arrival point

$T=$ time in hours from time of calculation to required ETA

$O=$ present speed ordered

$H=$ second, lower speed; that is, terminal speed.

Let us take an example. At 2030 the navigator is assessing what speed changes are required prior to his making the pilotage ground at 0900 the next morning. He wishes to finish the passage at 10 knots, this speed allowing economical running and safe working conditions on the upper deck. At the moment he has 18 knots rung on. Referring to chart or navigation system, he sees that he has 192 nautical miles to run from 2100 to his arrival point. Substituting these values into the formula gives:

$$
\begin{aligned}
& t=\frac{192-(10 \times 12)}{(18-10)} \\
& t=9 .
\end{aligned}
$$

This means that at $2100+9$ hours - that is, $0600-$ he can reduce speed to 10 knots.

Not only is this formula more blunder-resistant than the simultaneous equations of Captain Fales, it also allows easy and intuitive refinement. For example, the net effect of current and tidal stream is 3 miles advantageous, so $D$ is reduced by 3 nautical miles. The navigator, ever cautious, wants to keep half an hour in hand, so $T=(12-0.5)$. Those with real class will also allow for the time of gaining and losing speed : for example, a heavy unit may carry its way for Io minutes after reducing speed, allowed for by reducing $T$ by an equivalent amount. For the perverse navigator who wants to go slowly now and arrive at high speed, the formula is equally applicable.

The navigator can thus present this solution with the confidence that he has sidestepped blunder country, and can go to bed secure in the knowledge that the formula is simple enough for the overnight OOWs to check at frequent intervals, thus ensuring a painless and economical arrival. Almost total blunder-proofing can be applied by calculating the critical distance $D$ in different ways; for example, one working can be done using $D$ obtained from a nav system waypoint chain, and another check calculation can use $D$ obtained from a small-scale planning chart or the distances to go marked on the bridge charts. It is easy to judge whether a difference in answers is due to the 'slop' involved in manual chartwork, or whether a blunder has invited itself in.

Should there be a mismatch between bridge time and the time kept at the destination, the scope for mischief is reduced because all times are elapsed bridge times, other than the first calculation of total time available.

Success is therefore 99 percent certain, and the navigator need no longer shuffle around with compasses and furthest-on circles, knowing that he is in blunder country and will in any case get a less precise solution. 


\title{
REFERENCE
}

1 Fales, T. (1992). Assuring punctual arrival and concomitant economy of fuel. This Journal, 45, 134 .

KEY WORDS

I. Voyage planning. 2. Military navigation.

\section{'Navigation with $\mathrm{km}$ and gon'}

\author{
K. K. White
}

In an article published in the September 1991 edition of this Journal, ' S. Stubert put forward an interesting and informative case in support of the universal adoption of the gon (grade) for the measurement of angle and arc in navigation. However, while the gon is a decimal unit, and while it may be said that in general the introduction of simpler decimal measurements into navigation should be beneficial, this would only be the case if the gon system were the best possible. I am unable to agree that it is, for the four main reasons which follow.

First, the gon system does not meet the criteria for good decimal measurement which were outlined in an article entitled 'A decimal system of navigation' which was published in the May 1986 edition of Navigation News. Of particular note is the view that a good decimal system for measuring any kind of quantity should be based on one unit only and not on three as in the gon system, namely the circle, the right angle and the gon. Also, it may be noted that the division of the gon into a hundred parts or centigons does not conform with the preference of the International Metric System (SI) for decimal submultiples with prefixes such as milli- and micro- and not for submultiples prefixed by centi-, except in special circumstances.

Secondly, it would seem to be wrong to even consider the suitability of a decimal system for measuring angle and arc for the purposes of navigation without at the same time considering the suitability of a complementary system of measuring time, because the measurement of angle, arc and time are all closely related in navigation. It would appear from Stubert's article that the gon system lacks a complementary decimal timemeasuring system, and it is doubtful that one developed on similar lines to the gon involving a division of the day into quarters, followed by a division of each quarter-day into decimal fractions, would find general acceptance.

Thirdly, what is considered to be a better decimal system for measuring angle and arc has been developed and described in the article in Navigation News mentioned above. It is based on the circle itself, which is divided into decimal submultiples which are convenient and which conform with SI preferences such as the millicircle and microcircle. This is complemented by a decimal system of measuring time based on the day, which is likewise divided into convenient and preferred SI decimal submultiples such as the milliday and microday. This simple and coherent decimal system of measuring time, angle and arc, which has been called for brevity the 'decimal-day-circle' or DDC system offers, in theory at least, a superior measuring system to any other which is based purely on the decimalization of some non-decimal portion of a circle, like the gon or the degree, and lacks a complementary and coherent decimal time-measuring system. 